\title{
RETRACTED ARTICLE: Cotransplantation of human umbilical cord mesenchymal and haplo-hematopoietic stem cells in patients with severe aplastic anemia
}

\author{
Lixin Xu $\cdot$ Zhouyang Liu $\cdot$ Yamei Wu $\cdot$ Xueliang Yang $\cdot$ \\ Yongbin Cao $\cdot$ Xiaohong Li $\cdot$ Bei Yan $\cdot$ Songwei Li $\cdot$ \\ Wanming Da $\cdot$ Xiaoxiong Wu
}

Received: 22 June 2014/ Accepted: 27 September 2014/Published online: 17 August 2015

(C) Springer Science+Business Media Dordrecht 2014

The Publisher and Editor retract this article in accordance with the recommendations of the Committee on Publication Ethics (COPE). After a thorough investigation we have strong reason to believe that the peer review process was compromised.

The original article was published online on 19th November 2014.

L. Xu $\cdot$ Z. Liu $\cdot$ Y. Wu $\cdot$ X. Yang $\cdot$ Y. Cao

X. Li $\cdot$ B. Yan $\cdot$ S. Li $\cdot$ X. Wu $(\triangle)$

Department of Hematology, the First Affiliated Hospital of Chinese PLA General Hospital, No. 51 Fucheng Road, Haidian District, Beijing 100853, China

e-mail: wuxiaoxiong123@126.com

W. Da

Department of Hematology, Chinese PLA General

Hospital, Beijing 100048, China 